\title{
Structural Characterization of a Novel Two-Dimensional Material: Cobalt Sulfide Sheets on Au(111)
}

\author{
Mahesh K. Prabhu, ${ }^{\S}$ Dajo Boden, ${ }^{\S}$ Marcel J. Rost, Jörg Meyer,* and Irene M. N. Groot*
}

Cite This: J. Phys. Chem. Lett. 2020, 11, 9038-9044

Read Online

ABSTRACT: Transition metal dichalcogenides (TMDCs) are a type of two-dimensional (2D) material that has been widely investigated by both experimentalists and theoreticians because of their unique properties. In the case of cobalt sulfide, density functional theory (DFT) calculations on free-standing $\mathrm{S}-\mathrm{Co}-\mathrm{S}$ sheets suggest there are no stable $2 \mathrm{D}$ cobalt sulfide polymorphs, whereas experimental observations clearly show TMDC-like structures on $\mathrm{Au}(111)$. In this study, we resolve this disagreement by using a combination of experimental techniques and DFT calculations, considering the substrate explicitly. We find a 2D CoS(0001)-like sheet on $\mathrm{Au}(111)$ that delivers excellent agreement between theory and experiment. Uniquely this sheet exhibits a metallic character, contrary to most TMDCs, and exists due to the stabilizing interactions with the $\mathrm{Au}(111)$ substrate.

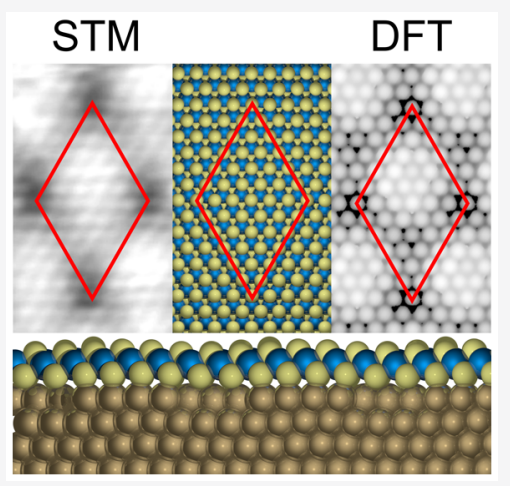

$\mathrm{T}$ ransition metal dichalcogenides (TMDCs) make up a class of two-dimensional (2D) materials that have been extensively researched over the past two decades because of their unique chemical and mechanical properties for applications in (opto)electronics, biology, high-density storage, efficient energy harvesting, high-resolution imaging, and catalysis. $^{1-7}$ The scientific community has been on a quest to discover new TMDCs with unique properties to improve existing applications as well as to find completely new ones. Currently, the metals of groups 4-6 and those of group 10 are being thoroughly investigated, because in the bulk they form layered TMDCs held together by van der Waals forces. ${ }^{8}$ The weak van der Waals forces enable mechanical or chemical exfoliation of $2 \mathrm{D}$ sheets from the bulk materials. ${ }^{9}$

Higher-quality single-layer TMDCs can also be synthesized by epitaxial growth on a suitable substrate, for example, singlelayer TMDCs of $\mathrm{Mo},{ }^{10} \mathrm{~W},{ }^{11} \mathrm{Ti}^{12}{ }^{12} \mathrm{Ta},{ }^{13} \mathrm{Zr},{ }^{14} \mathrm{Nb},{ }^{15}$ and $\mathrm{Hf}^{16}$ Gold substrates are a preferred choice for the growth of many of these single-layer TMDCs as the high chalcogen affinity of gold can have a stabilizing effect. ${ }^{17}$ Consequently, to properly investigate the existence and specific properties of potential TMDCs, interactions with the support must be considered.

Theoretical methods, such as density functional theory (DFT), are powerful tools for predicting new TMDC candidates with unique properties. However, the support is often not considered, which causes a major discrepancy between calculations and reality and limits the scope of the predictions. For instance, Ataca et al. ${ }^{18}$ investigated most of the transition metals for their ability to form TMDCs using freestanding sheets in vacuum. They found that cobalt sulfide does not form stable TMDC sheets. This stands in disagreement with some experimental work by Kibsgaard et al., ${ }^{19}$ who showed TMDC-like cobalt sulfide sheets are formed on $\mathrm{Au}(111)$ after exposing cobalt nanoparticles to $\mathrm{H}_{2} \mathrm{~S}$.

In this work, we demonstrate that, when including the $\mathrm{Au}(111)$ substrate explicitly in our DFT calculations, excellent agreement between experiment and theory can be achieved for $2 \mathrm{D}$ cobalt sulfide. We obtain an atomic model for the cobalt sulfide sheets that nicely fits with our experimental results from scanning tunneling microscopy (STM), low-energy electron diffraction (LEED), and X-ray photoelectron spectroscopy (XPS). Our structure, which is similar to that of bulkterminated $\operatorname{CoS}(0001)$, improves the model suggested by Kibsgaard et al. ${ }^{19}$ In addition, the methodology used in our research could potentially be applied to other earth-abundant transition metals such as $\mathrm{Fe}, \mathrm{Ni}$, and $\mathrm{Cu}$ to explore the existence of other novel $2 \mathrm{D}$ materials.

Figure 1a shows the STM image of large 2D cobalt sulfide sheets grown on $\mathrm{Au}(111)$. The terraces of $\mathrm{Au}(111)$ no longer show the typical herringbone reconstruction after exposure to $\mathrm{H}_{2} \mathrm{~S}$ (see the Supporting Information). An identical distorted herringbone reconstruction of $\mathrm{Au}(111)$ upon exposure to $\mathrm{H}_{2} \mathrm{~S}$ has been observed in the experiments of ref 19 and also in lowcoverage thiol ${ }^{20-22}$ and hexabromobenzene ${ }^{23}$ adsorption experiments performed by other research groups. Upon flash

Received: July 24, 2020

Accepted: September 28, 2020

Published: September 28, 2020 

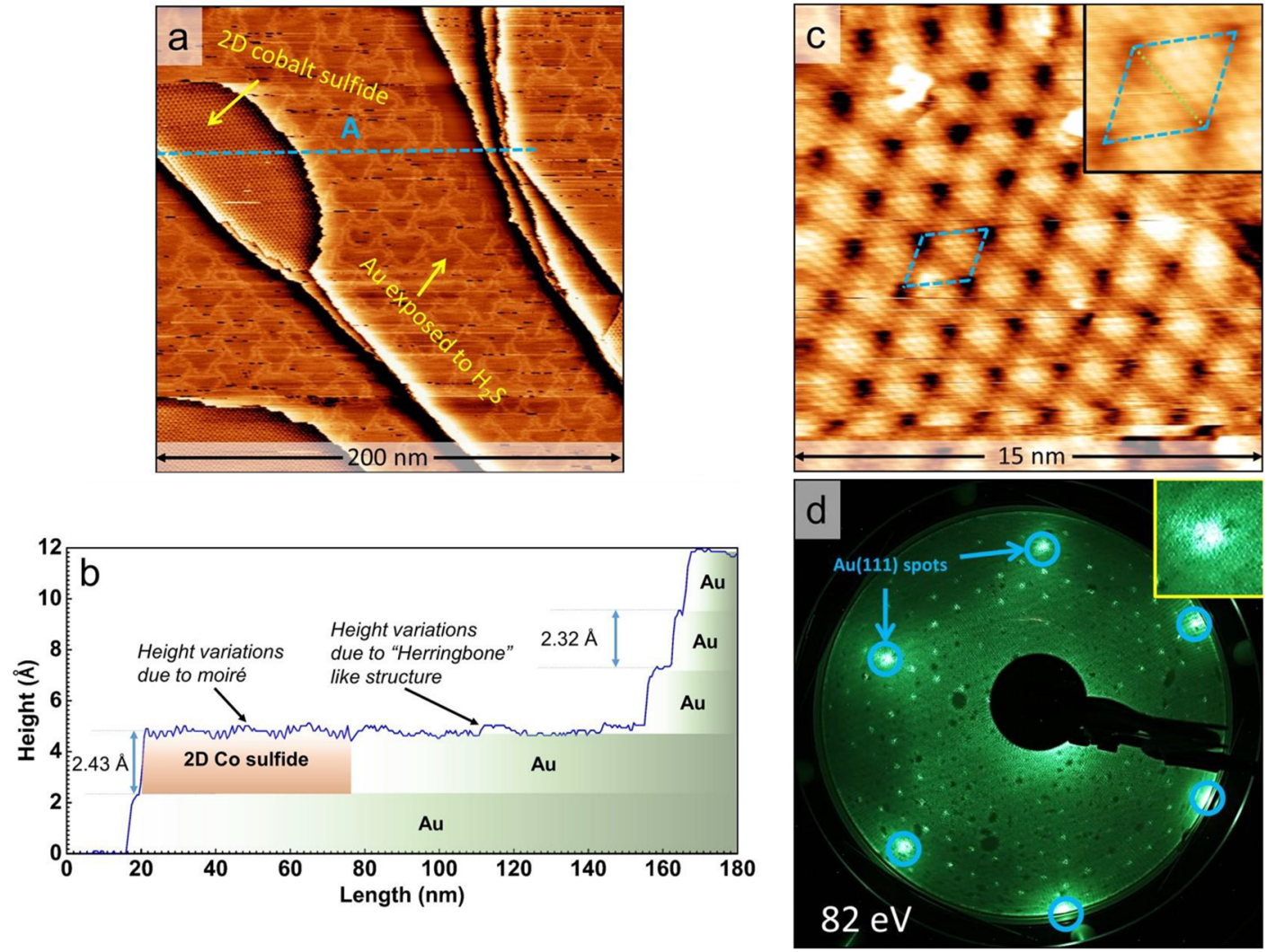

Figure 1. 2D cobalt sulfide supported on $\mathrm{Au}(111)$. (a) Large-scale STM image showing single-layer cobalt sulfide sheets. A modified reconstruction of $\mathrm{Au}(111)$ due to $\mathrm{H}_{2} \mathrm{~S}$ exposure with a long-range hexagonal arrangement is seen on the steps of gold. The respective phases are marked. (b) Measured height profile along the blue dashed line marked A in panel a. (c) Atomically resolved STM image of the 2D cobalt sulfide. The rhombus-shaped unit cell is outlined in blue with the two triangular halves demarcated by a green dotted line and enlarged in the inset for the sake of clarity. Image acquired at a sample voltage of $-0.7 \mathrm{~V}$. (d) LEED pattern of cobalt sulfide sheets on $\mathrm{Au}(111)$ with an incident energy of $95 \mathrm{eV}$. The diffraction spots of the $\mathrm{Au}(111)$ surface are indicated by blue circles. The inset shows a close-up of the Au spot that shows that the spots due to herringbone reconstruction are reduced to spot broadening.

annealing to $673 \mathrm{~K}$ in ultrahigh vacuum (UHV), the typical herringbone reconstruction is partially restored while the cobalt sulfide sheets remain intact (see the Supporting Information). Furthermore, height line A indicated in Figure $1 \mathrm{a}$ and shown in Figure $1 \mathrm{~b}$ reveals that the cobalt sulfide sheet has an average thickness of $2.43 \AA$ with corrugations of $\pm 0.2 \AA$ in comparison to an experimental $\mathrm{Au}-\mathrm{Au}$ step height of 2.32 Å.

The 2D cobalt sulfide grows as large pristine sheets into and out of the step edges of $\mathrm{Au}(111)$. The boundary line of the 2D cobalt sulfide sheets has a curved shape along the $\mathrm{Au}$-cobalt sulfide interface and the 2D cobalt sulfide step edge. Analysis of the contact angles between the line interfaces at the two triple points of the cobalt sulfide sheets reveals a mirror symmetry (see the Supporting Information). The corresponding triple points of different cobalt sulfide sheets, however, have surprisingly the same contact angles irrespective of the size of the sheets. This shows that the interfacial tension at the triple points of the interfaces is responsible for the observed shape of the cobalt sulfide islands. We think that the mirror symmetry arises from different edge terminations at the two triple points; however, further investigations into the interactions at the $\mathrm{Au}$ and $2 \mathrm{D}$ cobalt sulfide line interface are beyond the scope of this work.

The cobalt sulfide sheets have a hexagonal structure in their basal plane that has been further resolved in Figure 1c. This hexagonal structure consists of a rhombus-shaped unit cell marked in blue with six bright spots along its edges. This unit cell has a side length of $1.97 \mathrm{~nm}$, which corresponds to seven $\mathrm{Au}-\mathrm{Au}$ interatomic distances. We therefore classify this structure as a $7 \times 7$ cobalt sulfide structure with respect to $\mathrm{Au}(111)$. This $7 \times 7$ structure is further demonstrated by the LEED pattern of the cobalt sulfide sheets shown in Figure 1d. The bright spots encircled in blue arise from the bare $\mathrm{Au}(111)$ surface. The small satellite spots expected for $\mathrm{Au}(111)$ are reduced to spot broadening (see the inset of Figure 1d) due to the aforementioned modification of the herringbone reconstruction caused by the exposure to hydrogen sulfide. The smaller spots can be attributed to the Co sulfide sheets and show a hexagonal $7 \times 7$ symmetry with respect to the $\mathrm{Au}(111)$ spots in agreement with the STM results. The rhombus-shaped unit cell consists of two triangular halves, as indicated by a green dotted line in the inset of Figure 1c. One of the triangular halves (top right one) has a relative contrast that is higher than that of the other (bottom left one), causing the contrast to alternate in a hexagonal pattern. Additionally, the vertices of the rhombus-shaped unit cell appear dark in the STM image. Atomic resolution in Figure 1c reveals that the distances between the bright spots in the top layer of the cobalt sulfide of $\sim 3.3 \AA$ are similar to what was found by Kibsgaard et al., ${ }^{19}$ who attributed this pattern to a sheetlike structure similar to that of $\mathrm{Co}_{3} \mathrm{~S}_{4}(111)$.

To determine the stoichiometry of the cobalt sulfide sheets, the sample was flash annealed to $673 \mathrm{~K}$ in UHV to desorb the 

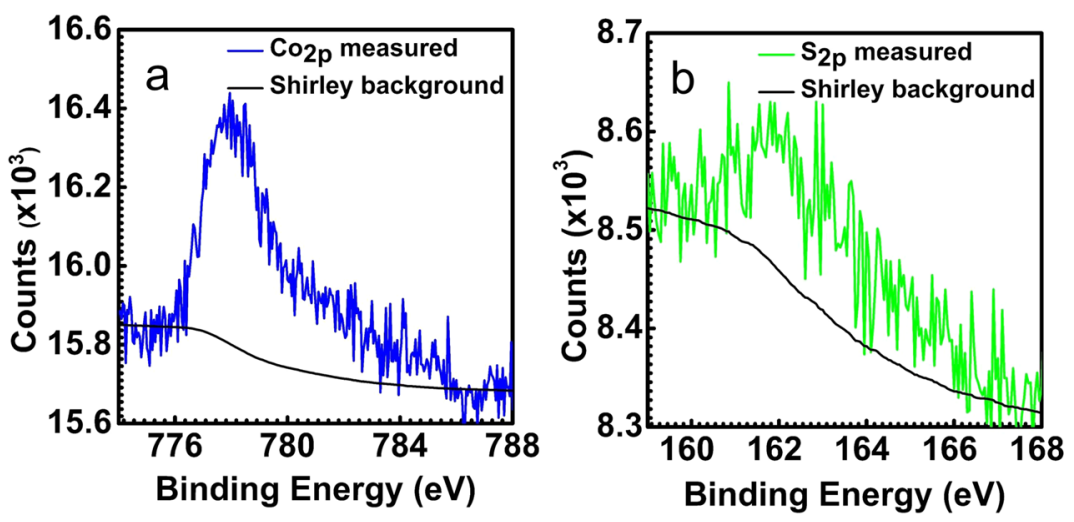

Figure 2. XPS spectra of $2 \mathrm{D}$ cobalt sulfide supported on $\mathrm{Au}(111)$ after flash annealing to $673 \mathrm{~K}$ to remove sulfur species adsorbed on the $\mathrm{Au}(111)$ terraces: (a) $\mathrm{Co}_{2 \mathrm{p}}$ and (b) $\mathrm{S}_{2 \mathrm{p}}$.

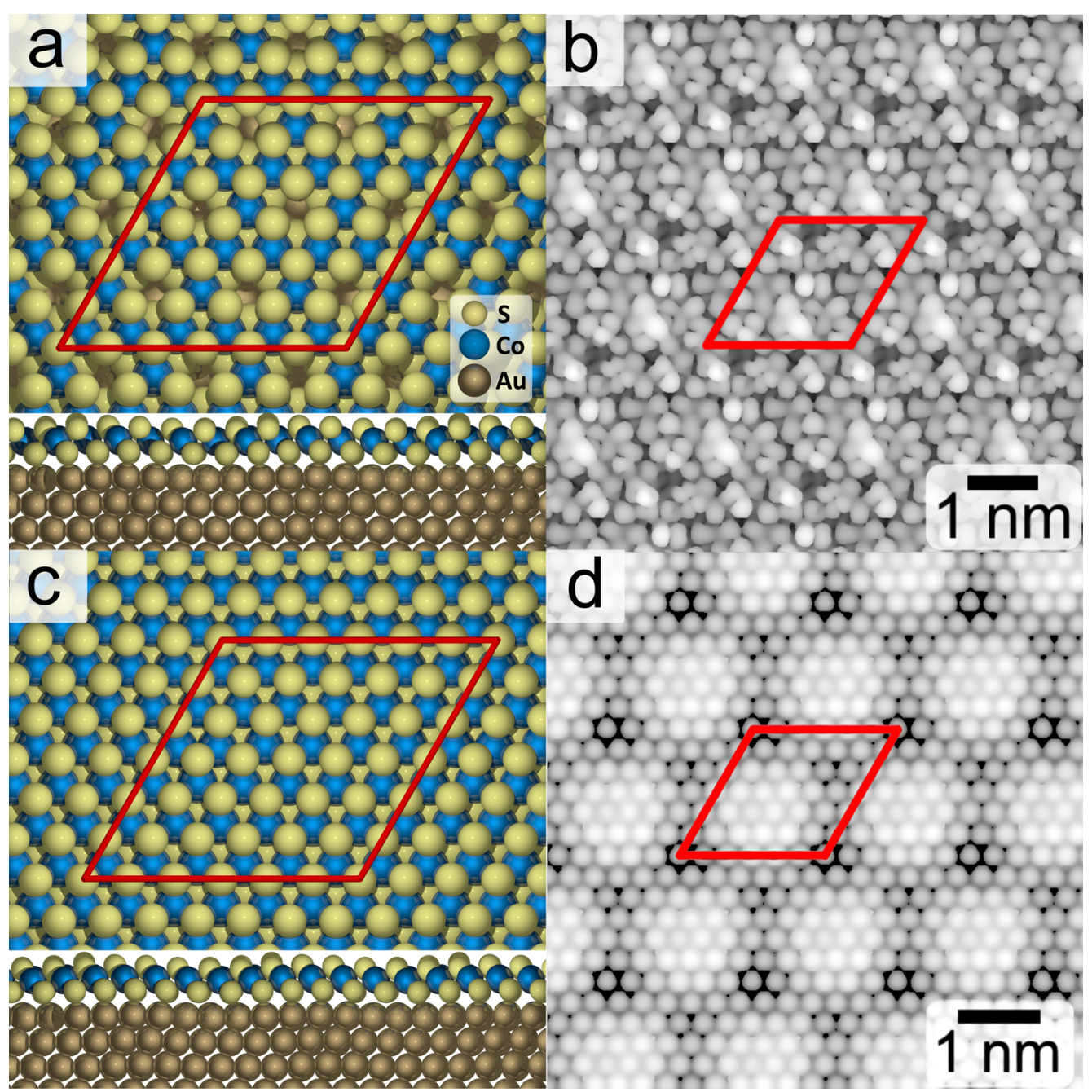

Figure 3. Schematic models of $7 \times 7$ overlayer structures on $\mathrm{Au}(111)$ comprised of (a) $\mathrm{Co}_{3} \mathrm{~S}_{4}(111)$-like and (c) $\mathrm{CoS}(0001)$-like $\mathrm{S}-\mathrm{Co}-\mathrm{S}$ sheets, each with a top (side) view in the top (lower) part of the image. (b and d) Simulated STM images of the structures resulting from relaxation of structures in panels a and c, respectively. Both images were simulated using the Tersoff-Hamann method with a bias potential of $-0.3 \mathrm{~V}$. The contrast has been adjusted for better comparison with Figure 1c. The unit cells in panels a-d are colored red.

sulfur species on the $\mathrm{Au}(111)$ terraces. After this step, XPS spectra were recorded (shown in Figure 2). The relative areas of the $\mathrm{Co}_{2 \mathrm{p}}$ and $\mathrm{S}_{2 \mathrm{p}}$ peaks were corrected by the surface relative sensitivity factors ${ }^{24}$ to determine the Co:S ratio. Our measurements show a Co:S ratio of 1:2.09 (see the Supporting Information for details), while an approximate Co:S ratio of
1:2.7 has been reported by Kibsgaard et al. ${ }^{19}$ using Auger electron spectroscopy.

Using the experimental findings in this work and that of Kibsgaard et al., ${ }^{19}$ we made use of DFT calculations to arrive at an atomic model. A logical first structure is a sheet based on $\mathrm{Co}_{3} \mathrm{~S}_{4}(111)$, as this is one of the most common bulk- 
terminated, hexagonal, cobalt sulfide surfaces reported in the literature. ${ }^{25}$ The experimental unit cell of $\mathrm{Co}_{3} \mathrm{~S}_{4}(111)$ is $6.65 \AA$ $\times 6.65 \AA$, and the lateral $\mathrm{S}-\mathrm{S}$ distance is $3.3 \AA$, which matches with the distance of $3.3 \AA$ between the bright spots in the STM image in Figure 1c. ${ }^{25}$ As discussed previously, the unit cell from LEED and STM corresponds to $7 \times 7$ gold atoms, which is only $\sim 1.3 \%$ smaller than the experimental size of $3 \times 3$ $\mathrm{Co}_{3} \mathrm{~S}_{4}(111)$ unit cells. To create a model for the cobalt sulfide sheet on gold, the top $\mathrm{S}-\mathrm{Co}-\mathrm{S}$ layers of a $3 \times 3 \mathrm{Co}_{3} \mathrm{~S}_{4}(111)$ supercell are fitted onto a $7 \times 7 \mathrm{Au}(111)$ surface. As shown in Figure $3 \mathrm{a}$, this results in a $\mathrm{S}-\mathrm{Co}-\mathrm{S}$ "sandwich" structure with a 3:8 Co:S stoichiometry that is very similar to what was suggested by Kibsgaard et al. ${ }^{19}$ However, this $\mathrm{Co}_{3} \mathrm{~S}_{4}(111)$-like structure proved to be unstable during relaxation with DFT. The simulated STM image of the resulting "rearranged" $\mathrm{Co}_{3} \mathrm{~S}_{4}(111)$-like sheet shown in Figure $3 \mathrm{~b}$ and the experimental STM image in Figure 1c showed no similarities. For details of the DFT relaxation, see the Supporting Information.

$\operatorname{CoS}(0001)$ is another bulk-terminated cobalt sulfide surface having a hexagonal unit cell with an experimental unit cell size of $3.38 \AA \times 3.38 \AA \AA^{26}$ This means a $6 \times 6$ supercell of bulkterminated $\operatorname{CoS}(0001)$ is $\sim 2.9 \%$ larger than the experimentally found unit cell of the cobalt sulfide sheet in Figure 1c. A schematic model of the $\mathrm{CoS}(0001)$-like sheet on $\mathrm{Au}(111)$ is shown in Figure 3c. It is very similar to the $\mathrm{Co}_{3} \mathrm{~S}_{4}(111)$-like sheet; however, there are no holes in the Co layer, resulting in a 1:2 Co:S stoichiometry. This matches the experimentally determined Co:S ratio of 1:2.09 more closely than that of the $\mathrm{Co}_{3} \mathrm{~S}_{4}(111)$-like sheet. Furthermore, the $\mathrm{CoS}(0001)$-like sheet remains stable during relaxation with DFT and the simulated STM image shown in Figure 3d reproduces most of the key features in the experimental STM image in Figure 1c. We note that the simulated STM images have a resolution significantly higher than what is achievable in an STM experiment because no tunneling integral is included accounting for the finite tip size. Comparison of the bond distances in Table 1 reveals that

Table 1. Average Bond Distances from the Structure Shown in Figure 3c after Relaxation Compared to Corresponding Bond Distances in Bulk Cobalt Sulfides and Thiols on $\operatorname{Au}(111)^{a}$

\begin{tabular}{|c|c|c|c|c|}
\hline structure & $d_{\mathrm{Au}-\mathrm{S}}(\AA)$ & $\begin{array}{c}d_{\text {Co-S(bottom) }} \\
(\AA)\end{array}$ & $\frac{d_{\text {Co-S(top) }}}{(\AA)}$ & $\begin{array}{c}d_{\mathrm{Co}-\mathrm{Co}} \\
(\AA)\end{array}$ \\
\hline $\begin{array}{l}\mathrm{CoS}(0001) \text {-like sheet/ } \\
\mathrm{Au}(111) \mathrm{T}\end{array}$ & 2.57 & 2.24 & 2.25 & 3.43 \\
\hline bulk $\mathrm{Co}_{3} \mathrm{~S}_{4}{ }^{25,27} \mathrm{E}$ & - & 2.27 & 2.27 & 3.33 \\
\hline bulk $\operatorname{CoS}^{26} \mathrm{E}$ & - & 2.33 & 2.33 & 3.37 \\
\hline thiols ${ }^{28,29} \mathrm{~T}$ & $2.63(0.18)$ & - & - & - \\
\hline
\end{tabular}

${ }^{a}$ Theoretically and experimentally obtained values are indicated with $\mathrm{T}$ and $\mathrm{E}$, respectively.

the bond distances within the $\operatorname{CoS}(0001)$-like sheet remain similar to experimental values found in both bulk $\mathrm{Co}_{3} \mathrm{~S}_{4}$ and $\mathrm{CoS}$ after relaxation with DFT, especially considering the known disparity stemming from the approximations in the DFT functional as discussed in Computational Details.

Because six lateral $S-S$ distances in the $\operatorname{CoS}(0001)$-like cobalt sulfide sheet are equivalent to seven $\mathrm{Au}-\mathrm{Au}$ distances along the $\mathrm{Au}(111)$ surface, a moiré pattern forms during relaxation with DFT featuring the striking bright and dark triangles seen in the STM images (Figure 1c), as well as the darker spots at the vertices. When the sulfur in the bottom layer sits close to the top of the underlying $\mathrm{Au}$ atom, it is slightly elevated $(\leq 0.5 \AA)$ compared to the sulfur atom near the hollow or bridge sites, as is clearly visible in Figure 4. This

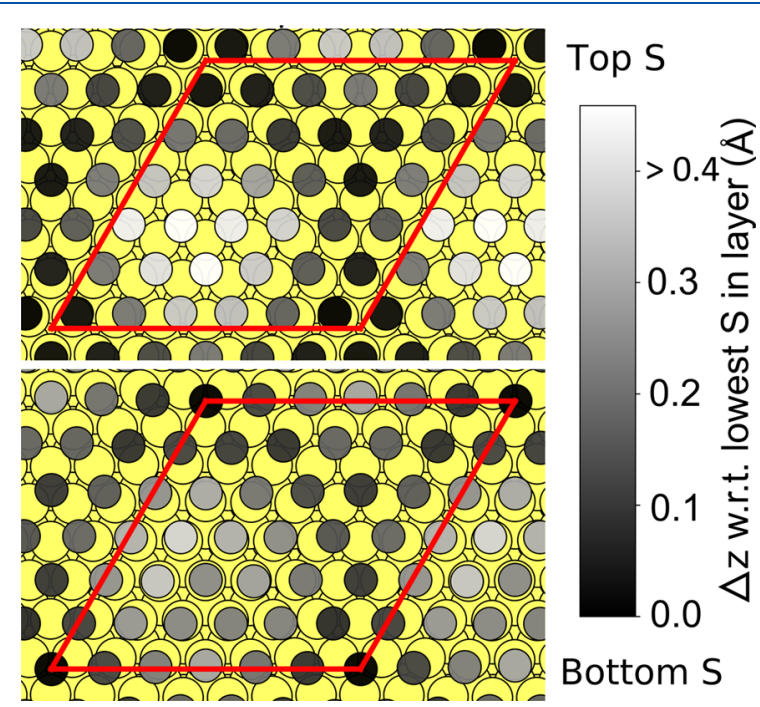

Figure 4. Positions of the sulfur atoms in the top and bottom basal planes of the cobalt sulfide sheet from Figure $3 \mathrm{c}$. The gold atoms are colored yellow, while the height of the sulfur atoms relative to the lowest atom in each respective layer is indicated in gray scale. The unit cell is displayed as a red rhombus. The height variation in the bottom layer of sulfur atoms due to the lattice mismatch with gold is exaggerated in the top layer. Comparison with Figure $3 \mathrm{~d}$ indicates the distinct pattern can be explained by height variations of atoms in the top layer, rather than any electronic effect.

causes a small corrugation in the entire cobalt sulfide sheet, which is exaggerated in the top sulfur layer. This leads to the brighter and darker areas in the STM images displayed in Figures $1 \mathrm{c}$ and $3 \mathrm{~d}$.

Unlike $\mathrm{MoS}_{2}$ and $\mathrm{TiS}_{2}$, no layered bulk structure has been reported for Co sulfide, whereby the interaction between layers consists of van der Waals forces only. The cobalt sulfide bulk structure that most closely resembles those of $\mathrm{MoS}_{2}$ and $\mathrm{TiS}_{2}$ is the previously discussed $\mathrm{CoS}$ bulk structure. However, single-layer cobalt sulfide sheets cannot be obtained by exfoliation of a CoS crystal, because the layers are held together by covalent bonds. ${ }^{17}$ Nevertheless, we demonstrate that $2 \mathrm{D}$ cobalt sulfide sheets can be synthesized on $\mathrm{Au}(111)$. Figure 5 shows that the interaction energy of the cobalt sulfide sheet with the $\mathrm{Au}(111)$ surface is $-0.40 \mathrm{eV}$ per sulfur atom in the bottom layer according to our DFT calculations. This is very similar to the strong $\mathrm{Au}-\mathrm{S}$ bond of thiols chemisorbed on $\mathrm{Au}(111),{ }^{28,29}$ as also further corroborated by the similarities in the bond lengths (see Table 1). The substantial interaction between gold and the cobalt sulfide sheet can also be discerned from the band structure and the (projected) densities of states available in the Supporting Information, which also reveals the metallic nature of the $\operatorname{CoS}(0001)$-like sheet. The formation of cobalt sulfide sheets on the $\mathrm{Au}(111)$ surface may be facilitated by the strong interaction between sulfur and gold, despite the presence of Co. This interaction appears to be covalent in nature, because there is almost no net charge transfer between the gold and the cobalst sulfide sheet (see the Supporting Information for more details). 


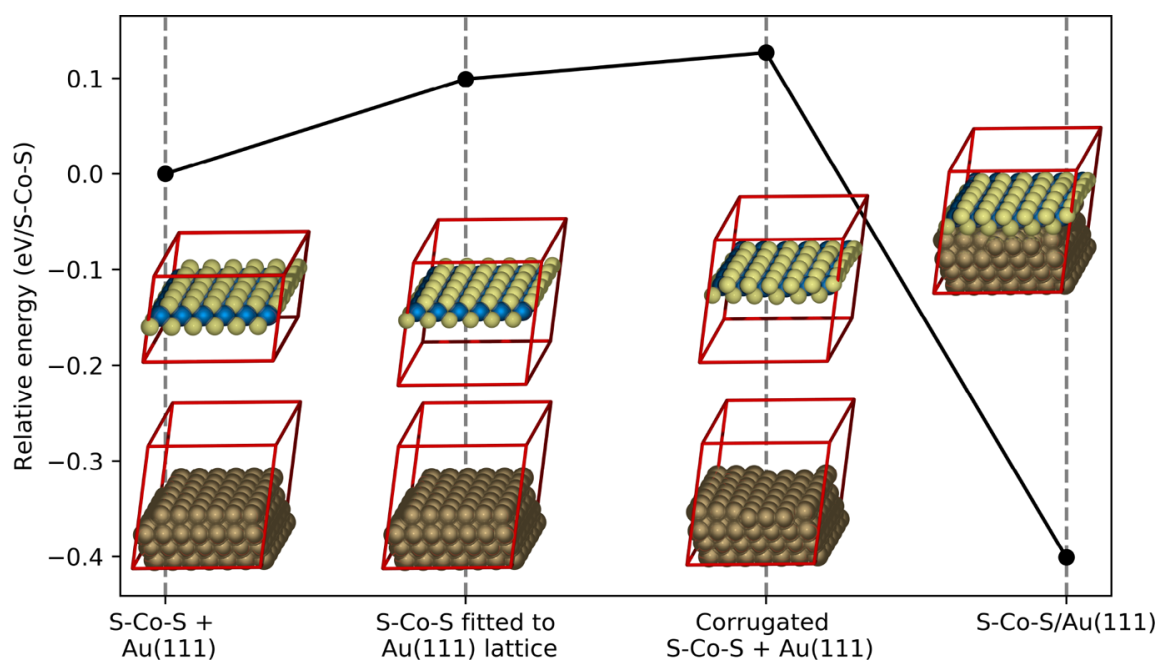

Figure 5. Decomposition of the interaction energy for the cobalt sulfide sheet and Au(111) as obtained from DFT calculations. Energies are per S$\mathrm{Co}-\mathrm{S}$ unit, with the separated $\mathrm{Au}(111)$ slab and free-standing $\mathrm{S}-\mathrm{Co}-\mathrm{S}$ film in equilibrium geometry shown in the leftmost inset defining zero energy. From left to right, the separated $\mathrm{Au}(111)$ slab and free-standing $\mathrm{S}-\mathrm{Co}-\mathrm{S}$ film compressed by $1 \%$ to coincide with ${ }^{7} /{ }_{6} \mathrm{Au}(111)$ surface lattice constants, same as before but additionally including the interfacial corrugation of the structure where the $\mathrm{S}-\mathrm{Co}-\mathrm{S}$ film and $\mathrm{Au}(111)$ are in contact, which is finally the case in the rightmost relaxed $7 \times 7 \mathrm{~S}-\mathrm{Co}-\mathrm{S}$ overlayer structure on $\mathrm{Au}(111)$ (i.e., same structure as schematically depicted in Figure 3c).

In summary, we show that $2 \mathrm{D}$ cobalt sulfide forms on $\mathrm{Au}(111)$ with a $\mathrm{CoS}(0001)$-like $\mathrm{S}-\mathrm{Co}-\mathrm{S}$ structure. The formation of $2 \mathrm{D}$ cobalt sulfide on $\mathrm{Au}(111)$ is facilitated by strong $\mathrm{Au}-\mathrm{S}$ interactions. The nature of the electronic interaction of cobalt sulfide with the $\mathrm{Au}(111)$ substrate is similar to that of $\mathrm{MoS}_{2}$; however, unlike $\mathrm{MoS}_{2}$, cobalt sulfide does not have a band gap. Because most TMDCs are semiconductors, cobalt sulfide stands out in this regard, which opens up possibilities for novel applications. In the case of $2 \mathrm{D}$ cobalt sulfide on $\mathrm{Au}(111)$, theory and experiment agree only when the substrate is considered explicitly in DFT calculations. This means that, when looking for potential new $2 \mathrm{D}$ materials, reliance solely on gas-phase calculations is not sufficient and a more complete approach is required.

\section{EXPERIMENTAL DETAILS}

All experiments were carried out in the ReactorSTM ${ }^{30}$ in UHV mode. $\mathrm{A} \mathrm{Au}(111)$ single crystal was prepared by repeated cycles of sputter cleaning with $\mathrm{Ar}^{+}$ions $(1.5 \mathrm{keV})$ and annealing to $873 \mathrm{~K}$. Two-dimensional cobalt sulfide was grown by evaporating cobalt in a $\mathrm{H}_{2} \mathrm{~S}$ atmosphere of $2 \times 10^{-6} \mathrm{mbar}$ onto the clean $\mathrm{Au}(111)$ single crystal maintained at $410 \mathrm{~K}$. Thereafter, the sample was annealed to $650 \mathrm{~K}$ for $20 \mathrm{~min}$ and cooled within $10 \mathrm{~min}$ to $473 \mathrm{~K}$ in the $\mathrm{H}_{2} \mathrm{~S}$ atmosphere. Lastly, the sample was cooled to $300 \mathrm{~K}$ within $120 \mathrm{~min}$ in UHV.

All STM imaging was performed with a tunneling current of $\sim 100 \mathrm{pA}$ and a sample voltage of $-1 \mathrm{~V}$ with the sample held at room temperature in UHV. To enhance the visibility of the details, we display the STM images with a line-by-line background subtraction. Note, however, that the height profiles are obtained from STM images with planar background filtering on the entire image ensuring a correctly connected 2D surface. All postprocessing is carried out using software developed in house (CAMERA version 4.3.5) as well as WSXM. ${ }^{31}$ LEED experiments were performed using a commercial Omicron SPECTALEED. The diffraction images were obtained with a Canon EOS50D camera using manual focusing and a $30 \mathrm{~s}$ exposure time. The LEED images shown here were contrast-enhanced further in RAW format without any other processing. The XPS spectra were measured using the SPECS-Phoibos system with a model HSA 3500 hemispherical analyzer. A monochromator was used to select $\mathrm{Al} \mathrm{K} \alpha$ radiation with a $1486.7 \mathrm{eV}$ excitation energy, and an incidence angle of $54.6^{\circ}$ was used. The spectra of $S_{2 p}$ and $\mathrm{Co}_{2 p}$ were recorded with a pass energy of $20 \mathrm{eV}$ and integrated 25 times to improve the signal-to-noise ratio. After acquisition, a Shirley background was subtracted using the CASA XPS software. To calculate the stoichiometry of $2 \mathrm{D}$ cobalt sulfide, the peak areas were corrected for their relative sensitivity factors. $^{24}$

\section{COMPUTATIONAL DETAILS}

All DFT calculations were performed with the Vienna $\mathrm{Ab}$

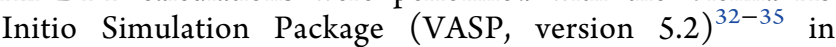
combination with the Atomic Simulation Environment (ASE, version 3.19$)^{36}$ for generating input and analyzing output files.

After having verified that spin polarization is not relevant for $\mathrm{CoS}$ (bulk structure) and $\mathrm{CoS}_{2}$ (bulk structure) consistent with earlier results, ${ }^{37}$ we used the non-spin-polarized formulation of the PBE exchange correlation functional ${ }^{38}$ for all of the surface calculations.

The Kohn-Sham orbitals were expanded into a plane-wave basis set with a cutoff energy of $300 \mathrm{eV}$, and ion-electron interactions were described by the projector-augmented wave method (PAW data set 52 from $2012^{39,40}$ ). Then, $12 \times 12 \times$ $12,7 \times 7 \times 7$, and $9 \times 9 \times 9$ Monkhorst-Pack k-point grids ${ }^{41}$ were used for the aforementioned $\mathrm{CoS}, \mathrm{CoS}_{2}$, and fcc-Au bulk structures, respectively, resulting in equilibrium lattice constants $a_{\mathrm{CoS}}^{\mathrm{DFT}}=3.35 \AA$ (with $\left.a_{\mathrm{CoS}}^{\mathrm{DFT}} / c_{\mathrm{CoS}}^{\mathrm{DFT}}=\frac{2}{3}\right), a_{\mathrm{CoS}_{2}}^{\mathrm{DFT}}=5.49$ $\AA$, and $a_{\mathrm{Au}}^{\mathrm{DFT}}=4.16 \AA$. This is in good agreement with the corresponding experimental values $a_{\mathrm{CoS}}^{\exp }=3.38 \AA$ (with $\left.a_{\mathrm{CoS}}^{\exp } / c_{\mathrm{CoS}}^{\exp }=\frac{2}{3}\right),{ }^{26} a_{\mathrm{CoS} S_{2}}^{\exp }=5.54 \AA \AA^{42}$ and $a_{\mathrm{Au}}^{\exp }=4.07 \AA{ }^{43}$ which is expected for the PBE functional. ${ }^{44}$ Despite this good agreement, differences between experimental and theoretical lengths remain; therefore, in this work, all distances discussed 
concern experimental values unless explicitly stated otherwise. Surface structures were based on a relaxed four-layer slab model for unreconstructed $\mathrm{Au}(111)$ with surface lattice constant $a_{\mathrm{Au}(111)}^{\mathrm{DFT}}=\frac{\sqrt{2}}{2} a_{\mathrm{Au}}^{\mathrm{DFT}}$. For the ensuing relaxations of the large-scale cobalt sulfide overlayer structures, the bottom three gold layers were frozen. For all of these calculations, a maximum force threshold of $10^{-3} \mathrm{eV} / \AA$ was used, together with a vacuum distance of $\geq 8 \AA$ and Monkhorst-Pack ${ }^{41} \mathrm{k}$ point grids corresponding to at least $13 \times 13 \times 1 \mathrm{k}$-points in the primitive $\mathrm{Au}(111)$ unit cell. STM images were simulated according to the Tersoff-Hamann approach ${ }^{45}$ as implemented in the HIVE-STM software package ${ }^{46}$ by plotting a surface of constant density of states within $0.05 \mathrm{eV}$ of the reported bias voltage.

\section{ASSOCIATED CONTENT}

\section{(s) Supporting Information}

The Supporting Information is available free of charge at https://pubs.acs.org/doi/10.1021/acs.jpclett.0c02268.

$\mathrm{Au}(111)$ exposed to $\mathrm{H}_{2} \mathrm{~S}, 2 \mathrm{D}$ cobalt sulfide sheets of various sizes supported on $\mathrm{Au}(111)$, XPS spectra, TMDC-like structure in sulfur-terminated $\mathrm{Co}_{3} \mathrm{~S}_{4}(111)$, TMDC-like structure in $\mathrm{CoS}(0001)$, density of states and band structure of $\mathrm{CoS}(0001) / \mathrm{Au}(111)$ from DFT, charge analysis, and van der Waals corrections (PDF)

Movie S1 (MP4)

Movie S2 (MP4)

\section{AUTHOR INFORMATION}

\section{Corresponding Authors}

Jörg Meyer - Leiden Institute of Chemistry, Leiden University, 2300 RA Leiden, The Netherlands; 이이이.org/0000-00030146-730X; Phone: +31 (0)71 527 5569; Email: j.meyer@ chem.leidenuniv.nl

Irene M. N. Groot - Leiden Institute of Chemistry, Leiden University, 2300 RA Leiden, The Netherlands; $\odot$ orcid.org/ 0000-0001-9747-3522; Phone: +31 (0)71 527 7361;

Email: i.m.n.groot@lic.leidenuniv.nl

\section{Authors}

Mahesh K. Prabhu - Leiden Institute of Chemistry, Leiden University, 2300 RA Leiden, The Netherlands

Dajo Boden - Leiden Institute of Chemistry, Leiden University, 2300 RA Leiden, The Netherlands

Marcel J. Rost - Huygens-Kamerlingh Onnes Laboratory, Leiden Institute of Physics, Leiden University, 2300 RA Leiden, The Netherlands

Complete contact information is available at:

https://pubs.acs.org/10.1021/acs.jpclett.0c02268

\section{Author Contributions}

${ }^{\S}$ M.K.P. and D.B. contributed equally to this work.

\section{Notes}

The authors declare no competing financial interest.

\section{ACKNOWLEDGMENTS}

The authors thank the Dutch Organization for Scientific Research (NWO) for providing computing time on the Cartesius facility (Grant 17216) and the Leiden Institute of Chemistry (LIC) for financial support.

\section{REFERENCES}

(1) Jariwala, D.; Sangwan, V. K.; Lauhon, L. J.; Marks, T. J.; Hersam, M. C. Emerging Device Applications for Semiconducting Twodimensional Transition Metal Dichalcogenides. ACS Nano 2014, 8, $1102-1120$.

(2) Chhowalla, M.; Shin, H. S.; Eda, G.; Li, L.-J.; Loh, K. P.; Zhang, H. The Chemistry of Two-dimensional Layered Transition Metal Dichalcogenide Nanosheets. Nat. Chem. 2013, 5, 263-275.

(3) Geim, A. K.; Grigorieva, I. V. Van Der Waals Heterostructures. Nature 2013, 499, 419-425.

(4) Lightcap, I. V.; Kosel, T. H.; Kamat, P. V. Anchoring Semiconductor and Metal Nanoparticles on a Two-dimensional Catalyst Material Storing and Shuttling Electrons with Reduced Graphene Oxide. Nano Lett. 2010, 10, 577-583.

(5) Cao, X. H.; Yin, Z. Y.; Zhang, H. Three-dimensional Graphene Materials: Preparation, Structures and Application in Supercapacitors. Energy Environ. Sci. 2014, 7, 1850-1865.

(6) Yin, Z. Y.; Zhu, J. X.; He, Q. Y.; Cao, X. H.; Tan, C. L.; Chen, H. Y.; Yan, Q. Y.; Zhang, H. Graphene-based Materials for Solar Cell Applications. Adv. Energy Mater. 2014, 4, 1300574.

(7) Gong, Y. J.; Lin, J. H.; Wang, X. L.; Shi, G.; Lei, S. D.; Lin, Z.; Zou, X. L.; Ye, G. L.; Vajtai, R.; Yakobson, B. I.; et al. Vertical and Inplane Heterostructures from $\mathrm{WS}_{2} / \mathrm{MoS}_{2}$ Monolayers. Nat. Mater. 2014, 13, 1135-1142.

(8) Manzeli, S.; Ovchinnikov, D.; Pasquier, D.; Yazyev, O. V.; Kis, A. 2D Transition Metal Dichalcogenides. Nat. Rev. Mater. 2017, 2, 17033.

(9) Geim, A. K. Random Walk to Graphene (Nobel Lecture). Angew. Chem., Int. Ed. 2011, 50, 6966-6985.

(10) Gronborg, S. S.; Ulstrup, S.; Bianchi, M.; Dendzik, M.; Sanders, C. E.; Lauritsen, J. V.; Hofmann, P.; Miwa, J. A. Synthesis of Epitaxial Single-layer $\mathrm{MoS}_{2}$ on $\mathrm{Au}(111)$. Langmuir 2015, 31, 9700-9706.

(11) Dendzik, M.; Michiardi, M.; Sanders, C.; Bianchi, M.; Miwa, J. A.; Gronborg, S. S.; Lauritsen, J. V.; Bruix, A.; Hammer, B.; Hofmann, P. Growth and Electronic Structure of Epitaxial Single-layer $\mathrm{WS}_{2}$ on $\mathrm{Au}(111)$. Phys. Rev. B: Condens. Matter Mater. Phys. 2015, 92, 245442.

(12) Biener, M. M.; Biener, J.; Friend, C. M. Novel Synthesis of Two-dimensional $\mathrm{TiS}_{2}$ Nanocrystallites on $\mathrm{Au}(111)$. J. Chem. Phys. 2005, 122, 034706.

(13) Kolekar, S.; Bonilla, M.; Ma, Y. J.; Diaz, H. C.; Batzill, M. Layer- and Substrate-dependent Charge Density Wave Criticality in 1T-TiSe 2 . 2D Mater. 2018, 5, 015006.

(14) Tsipas, P.; Tsoutsou, D.; Fragkos, S.; Sant, R.; Alvarez, C.; Okuno, H.; Renaud, G.; Alcotte, R.; Baron, T.; Dimoulas, A. Massless Dirac Fermions in $\mathrm{ZrTe}_{2}$ Semimetal Grown on InAs(111) by Van Der Waals Epitaxy. ACS Nano 2018, 12, 1696-1703.

(15) Stan, R. M.; Mahatha, S. K.; Bianchi, M.; Sanders, C. E.; Curcio, D.; Hofmann, P.; Miwa, J. A. Epitaxial Single-layer $\mathrm{NbS}_{2}$ on $\mathrm{Au}(111)$ : Synthesis, Structure, and Electronic Properties. Phys. Rev. Mater. 2019, 3, 044003.

(16) Tsoutsou, D.; Aretouli, K. E.; Tsipas, P.; Marquez-Velasco, J.; Xenogiannopoulou, E.; Kelaidis, N.; Aminalragia Giamini, S.; Dimoulas, A. Epitaxial 2D $\mathrm{MoSe}_{2}$ ( $\mathrm{HfSe}_{2}$ ) Semiconductor/2D $\mathrm{TaSe}_{2}$ Metal Van Der Waals Heterostructures. ACS Appl. Mater. Interfaces 2016, 8, 1836-1841.

(17) Sørensen, S. G.; Füchtbauer, H. G.; Tuxen, A. K.; Walton, A. S.; Lauritsen, J. V. Structure and Electronic Properties of In Situ Synthesized Single-layer $\mathrm{MoS}_{2}$ on a Gold Surface. ACS Nano 2014, 8, 6788-6796.

(18) Ataca, C.; Şahin, H.; Ciraci, S. Stable, Single-layer $\mathrm{MX}_{2}$ Transition-metal Oxides and Dichalcogenides in a Honeycomb-like Structure. J. Phys. Chem. C 2012, 116, 8983-8999.

(19) Kibsgaard, J.; Morgenstern, K.; Lægsgaard, E.; Lauritsen, J. V.; Besenbacher, F. Restructuring of Cobalt Nanoparticles Induced by Formation and Diffusion of Monodisperse Metal-sulfur Complexes. Phys. Rev. Lett. 2008, 100, 116104.

(20) Fitts, W. P.; White, J. M.; Poirier, G. E. Low-coverage Decanethiolate Structure on $\mathrm{Au}(111)$ : Substrate Effects. Langmuir 2002, 18, 1561-1566. 
(21) Liu, Y.-F.; Yang, Y.-C.; Lee, Y.-L. Assembly Behavior and Monolayer Characteristics of $\mathrm{OH}$-terminated Alkanethiol on $\mathrm{Au}(111)$ : In Situ Scanning Tunneling Microscopy and Electrochemical Studies. Nanotechnology 2008, 19, 065609.

(22) Darling, S. B.; Rosenbaum, A. W.; Wang, Y.; Sibener, S. J. Coexistence of the $(23 \times \sqrt{ } 3) \mathrm{Au}(111)$ Reconstruction and a Striped Phase Self-assembled Monolayer. Langmuir 2002, 18, 7462-7468.

(23) Huang, H.; Tan, Z.; He, Y.; Liu, J.; Sun, J.; Zhao, K.; Zhou, Z.; Tian, G.; Wong, S. L.; Wee, A. T. S. Competition Between Hexagonal and Tetragonal Hexabromobenzene Packing on $\mathrm{Au}(111)$. ACS Nano 2016, 10, 3198-3205.

(24) Wagner, C. D.; Davis, L. E.; Zeller, M. V.; Taylor, J. A.; Raymond, R. H.; Gale, L. H. Empirical Atomic Sensitivity Factors for Quantitative Analysis by Electron Spectroscopy for Chemical Analysis. Surf. Interface Anal. 1981, 3, 211-225.

(25) Lundqvist, D.; Westgren, A. Rontgenuntersuchung Des Systems Co-S. Z. Anorg. Allg. Chem. 1938, 239, 85-88.

(26) Alsén, N. Röntgenographische Untersuchung Der Kristallstrukturen Von Magnetkies, Breithauptit, Pentlandit, Millerit Und Verwandten Verbindungen. Geol. Foeren. Stockholm Foerh. 1925, 47, 19-72.

(27) Knop, O.; Reid, K. I. G.; Sutarno; Nakagawa, Y. Chalkogenides of the Transition Elements. VI. X-ray, Neutron, and Magnetic Investigation of the Spinels $\mathrm{Co}_{3} \mathrm{O}_{4}, \mathrm{NiCo}_{2} \mathrm{O}_{4}, \mathrm{Co}_{3} \mathrm{~S}_{4}$, and $\mathrm{NiCo}_{2} \mathrm{~S}_{4}$. Can. J. Chem. 1968, 46, 3463-3476.

(28) Franke, A.; Pehlke, E. Adsorption and Diffusion of $\mathrm{SCH}_{3}$ Radicals and $\mathrm{Au}\left(\mathrm{SCH}_{3}\right)_{2}$ Complexes on the Unreconstructed $\mathrm{Au}(111)$ Surface in the Submonolayer Coverage Regime. Phys. Rev. B: Condens. Matter Mater. Phys. 2009, 79, 44-46.

(29) Franke, A.; Pehlke, E. First-principles Study of 1,4butanedithiol Molecules and Radicals Adsorbed on Unreconstructed $\mathrm{Au}(111)$ and $\mathrm{Au}(100)$. Phys. Rev. B: Condens. Matter Mater. Phys. 2010, 81, 075409.

(30) Herbschleb, C. T.; van der Tuijn, P. C.; Roobol, S. B.; Navarro, V.; Bakker, J. W.; Liu, Q.; Stoltz, D.; Cañas-Ventura, M. E.; Verdoes, G.; van Spronsen, M. A.; et al. The ReactorSTM: Atomically Resolved Scanning Tunneling Microscopy Under High-pressure, High-temperature Catalytic Reaction Conditions. Rev. Sci. Instrum. 2014, 85, 083703.

(31) Horcas, I.; Fernández, R.; Gómez-Rodríguez, J. M.; Colchero, J.; Gómez-Herrero, J.; Baro, A. M. WSXM: A Software for Scanning Probe Microscopy and a Tool for Nanotechnology. Rev. Sci. Instrum. 2007, 78, 013705.

(32) Kresse, G.; Hafner, J. Ab Initio Molecular Dynamics for Liquid Metals. Phys. Rev. B: Condens. Matter Mater. Phys. 1993, 47, 558-561.

(33) Kresse, G.; Hafner, J. Ab-initio Molecular-dynamics Simulation of the Liquid-metal Amorphous-semiconductor Transition in Germanium. Phys. Rev. B: Condens. Matter Mater. Phys. 1994, 49, 14251-14269.

(34) Kresse, G.; Furthmüller, J. Efficiency of Ab-initio Total Energy Calculations for Metals and Semiconductors Using a Plane-wave Basis Set. Comput. Mater. Sci. 1996, 6, 15-50.

(35) Kresse, G.; Furthmüller, J. Efficient Iterative Schemes for Ab Initio Total-energy Calculations Using a Plane-wave Basis Set. Phys. Rev. B: Condens. Matter Mater. Phys. 1996, 54, 11169-11186.

(36) Hjorth Larsen, A.; Jørgen Mortensen, J.; Blomqvist, J.; Castelli, I. E.; Christensen, R.; Dułak, M.; Friis, J.; Groves, M. N.; Hammer, B.; Hargus, C.; et al. The Atomic Simulation Environment-a Python Library for Working with Atoms. J. Phys.: Condens. Matter 2017, 29, 273002.

(37) Feng, Z. Y.; Yang, Y.; Zhang, J. M. The Structural, Electronic and Magnetic Properties of $\mathrm{CoS}_{2}$ Under Pressure. Solid State Commun. 2018, 273, 60-65.

(38) Perdew, J. P.; Burke, K.; Ernzerhof, M. Generalized Gradient Approximation Made Simple. Phys. Rev. Lett. 1996, 77, 3865-3868.

(39) Blöchl, P. E. Projector Augmented-wave Method. Phys. Rev. B: Condens. Matter Mater. Phys. 1994, 50, 17953-17979.
(40) Kresse, G.; Joubert, D. From Ultrasoft Pseudopotentials to the Projector Augmented-wave Method. Phys. Rev. B: Condens. Matter Mater. Phys. 1999, 59, 1758-1775.

(41) Monkhorst, H. J.; Pack, J. D. Special Points for Brillouin-zone Integrations. Phys. Rev. B 1976, 13, 5188-5192.

(42) Nowack, E.; Schwarzenbach, D.; Hahn, T. Charge Densities in $\mathrm{CoS}_{2}$ and $\mathrm{NiS}_{2}$ (pyrite structure). Acta Crystallogr., Sect. B: Struct. Sci. 1991, 47, 650-659.

(43) Davey, W. P. Precision Measurements of the Lattice Constants of Twelve Common Metals. Phys. Rev. 1925, 25, 753-761.

(44) Schimka, L.; Gaudoin, R.; Klimes; Marsman, M.; Kresse, G. Lattice Constants and Cohesive Energies of Alkali, Alkaline-earth, and Transition Metals: Random Phase Approximation and Density Functional Theory Results. Phys. Rev. B: Condens. Matter Mater. Phys. 2013, 87, 214102.

(45) Tersoff, J.; Hamann, D. R. Theory of the Scanning Tunneling Microscope. Phys. Rev. B: Condens. Matter Mater. Phys. 1985, 31, 805-813.

(46) Vanpoucke, D. E. P.; Brocks, G. Formation of Pt-induced Ge Atomic Nanowires on $\mathrm{Pt} / \mathrm{Ge}(001)$ : A Density Functional Theory Study. Phys. Rev. B: Condens. Matter Mater. Phys. 2008, 77, 241308. 